\title{
Phase-field model for multiphase systems with preserved volume fractions
}

\author{
Britta Nestler, Frank Wendler, and Michael Selzer \\ Institute of Computational Engineering, Karlsruhe University of Applied Sciences, Moltkestrasse 30, D-76133 Karlsruhe, Germany \\ Björn Stinner and Harald Garcke \\ NWF I-Mathematik, Universität Regensburg, D-93040 Regensburg, Germany \\ (Received 2 January 2008; revised manuscript received 10 June 2008; published 28 July 2008)
}

\begin{abstract}
We report on an interesting formulation of a phase-field model which incorporates a description of individual phases and particles with preserved volume evolving in a system of multiple phases such that the interfacial energy decreases. In our model, an antiforcing free energy density is defined to fulfill constraints on selected volume fractions by counterbalancing phase changes. Phases are defined as regions with energy bearing boundaries that may differ in their physical states, i.e., the regions may be distinguished in structure (crystal transformations), in composition (alloys, mixtures of fluids), or in the orientation of the crystal lattice (grains). The method allows one to simulate the formation of equilibrium crystal shapes and of the migration of inert particles and phases in microstructures. We show two- and three-dimensional simulations of bubble ensembles and foam textures and demonstrate the excellent agreement of crystal morphology configurations with analytical results.
\end{abstract}

DOI: 10.1103/PhysRevE.78.011604

\section{INTRODUCTION}

Inhomogeneous systems characterized by multiple regions of different physical states, which will be called phases in the following, frequently involve energy contributions located along the phase interfaces. In this paper, we present a phase-field model that allows us to study the evolution of multiphase systems in which the interfacial energy decreases and some of the phases obey volume constraints, i.e., on the time scale of the interface motion, volume changes of those phases due to more or less complex physical processes can be neglected.

Applications of models for systems with a conserved volume for all constituents (cells, bubbles, or grains) range from foam structures and bubble clusters [1-3] to crystal growth with surface attachment limited kinetics [4] and morphologic stability of double crystals [5]. On the other hand, there are various systems driven by the reduction of surface energy, in which only part of the phases involved are subject to a constant volume. These can be found in the coarsening of polycrystals in contact with inert particles or second-phase grains $[6,7]$ or in equilibrium with a melt phase [8]. The presented model is based on a previously developed phase-field model [9] for multiphase systems. For a set of order parameters, each one representing a particular phase, the evolution is given as a gradient flow dynamics of the interfacial energy. For the latter one the Ginzburg-Landau approach has been used to state it in terms of the order parameters. To take volume constraints of some of the phases into account, the idea is to add a so-called "redistribution energy" to the system energy, whence additional forcing terms appear in the evolution equation. The new energy contribution involves time dependent coefficients that have to (and indeed can; we will state explicit formulas) be adaptively chosen in such a way that the volume constraints are fulfilled during the evolution. In some sense, these new forces act in the opposite direction of the interfacial forces arising from the GinzburgLandau energy and hence may be considered as antiforces.
PACS number(s): 81.10.Aj, 05.70.Ln, 47.55.db, 47.57.Bc

The Ginzburg-Landau energy consists of a gradient potential and a multiwell potential. Multiwell potentials of obstacle type revealed to be advantageous with respect to the calibration of surface energy densities $[10,11]$ but lead to variational inequalities. They therefore require a special numerical treatment as, e.g., in [12]. In [13], a model with volume preserving Allen-Cahn type phase equations coupled with Navier-Stokes type linear momentum equations is presented and applications to drop formation processes are shown.

The paper is organized as follows: In Sec. II A, we subsume all relevant details of the multiphase-field model including the different contributions to the free energy of the system. An extension of the model including the volume conservation of arbitrary phase-field parameters is given in Sec. II B. Starting from the formulation of the evolution equations, a nonlocal term for the bulk free energy is derived, which reconstitutes the volume changes during the evolution. In Sec. III, we present an effective numerical method to solve the evolution equations for the phase fields. As concrete examples for the application of the new model, simulations of bubble ensembles, double crystals, and foam structures are carried out and the results discussed in Sec. IV.

\section{PHASE-FIELD MODEL FOR MULTIPHASE SYSTEMS}

\section{A. Basic multiphase-field model}

We consider a general system of $N$ phases, i.e., of $N$ regions with energy bearing boundaries that may differ in their physical states. The different physical states can represent either different structures, compositions, or orientations of the crystal lattice. To model multiphase systems, we introduce a vector-valued continuous order parameter $\phi(\vec{x}, t)$ $=\left(\phi_{1}(\vec{x}, t), \ldots, \phi_{N}(\vec{x}, t)\right)$ where each component $\phi_{\alpha}(\vec{x}, t)$ of the vector describes the state of phase $\alpha$ and depends on a three-dimensional spatial coordinate $\vec{x}$ and on the time $t$. The 
formulation of the phase-field model for multiple order parameters is based on a Ginzburg-Landau energy density functional of the form

$$
\mathcal{F}(\phi)=\int_{\Omega}\left(\varepsilon a(\phi, \nabla \phi)+\frac{1}{\varepsilon} w(\phi)+f(\phi)\right) d x,
$$

where $\Omega$ is the domain of consideration and $\varepsilon$ defines the thickness of the diffuse interface in which the order param- eters $\phi_{\alpha}(\vec{x}, t)$ smoothly vary between two different physical states $\phi_{\alpha}(\vec{x}, t)=1$ and $\phi_{\alpha}(\vec{x}, t)=0$.

As described in [10], the dynamical equations for the evolution of $\phi_{\alpha}$ (the phase-field equations) are derived from the Ginzburg-Landau functional $\mathcal{F}(\phi)$ in Eq. (1) as variational derivatives with respect to the order parameters $\phi_{\alpha}$. In full generality they read as follows where we additionally introduce by $\mathrm{rhs}_{\alpha}$ a short notation:

$$
\tau \varepsilon \partial_{t} \phi_{\alpha}=\underbrace{\varepsilon\left[\nabla \cdot a_{, \nabla \phi_{\alpha}}(\phi, \nabla \phi)-a_{, \phi_{\alpha}}(\phi, \nabla \phi)\right]-\frac{1}{\varepsilon} w_{, \phi_{\alpha}}(\phi)-f_{,_{\phi}}(\phi)}_{=: \mathrm{rhs}_{\alpha}}-\lambda, \quad \alpha=1, \ldots, N .
$$

The parameter $\tau$ is a constant isotropic kinetic coefficient that may depend, in a general anisotropic system, on the orientation of the interface. The notation $a_{, \nabla \phi_{\alpha}}(\phi, \nabla \phi)$, $a_{, \phi_{\alpha}}(\phi, \nabla \phi), w_{, \phi_{\alpha}}$, and $f_{,_{\phi}}(\phi)$ is used to indicate the partial derivatives $\partial / \partial\left(\nabla \phi_{\alpha}\right)$ and $\partial / \partial \phi_{\alpha}$ of the functions $a(\phi, \nabla \phi)$, $w(\phi)$, and $f(\phi)$, respectively. The time derivative $\partial \phi_{\alpha}(\vec{x}, t) / \partial t$ is indicated by $\partial_{t} \phi_{\alpha}$ and $\nabla \cdot()$ denotes the divergence of a vector field $a_{, \nabla \phi_{\alpha}}(\phi, \nabla \phi)$. The Lagrange multiplier $\lambda$ is defined such that the constraint $\sum_{\alpha=1}^{N} \phi_{\alpha}(\vec{x}, t)=1$ is ensured, i.e.,

$$
\lambda=\frac{1}{N} \sum_{\alpha=1}^{N} \mathrm{rhs}_{\alpha}
$$

In the following, we give examples for functional expressions of the three types of energy density contributions. The gradient energy density $a(\phi, \nabla \phi)$ can be formulated in terms of a generalized gradient vector $q_{\alpha \beta}=\phi_{\alpha} \nabla \phi_{\beta}-\phi_{\beta} \nabla \phi_{\alpha}$ by

$$
a(\phi, \nabla \phi)=\sum_{\alpha<\beta} \gamma_{\alpha \beta}\left|\phi_{\alpha} \nabla \phi_{\beta}-\phi_{\beta} \nabla \phi_{\alpha}\right|^{2}
$$

For simplicity, we assume the surface energy density $\gamma_{\alpha \beta}$ of the $\alpha / \beta$ boundary to be isotropic. The gradient vector $q_{\alpha \beta}$ is oriented in normal direction to the interface. Anisotropy of the surface energy can be introduced into the model by letting $\gamma_{\alpha \beta}$ depend on $q_{\alpha \beta}$ (see [10] for examples of functional expressions). The potential part $w(\phi)$ of the energy density functional is assumed to be a multiobstacle type potential with higher order terms,

$$
w(\phi)=\frac{16}{\pi^{2}} \sum_{\alpha<\beta} \gamma_{\alpha \beta} \phi_{\alpha} \phi_{\beta}+\sum_{\alpha<\beta<\delta} \gamma_{\alpha \beta \delta} \phi_{\alpha} \phi_{\beta} \phi_{\delta} .
$$

The higher order terms $\sim \phi_{\alpha} \phi_{\beta} \phi_{\gamma}$ avoid the effect of ghost phase occurences at interfaces between two physical states. Further, we define free energy densities for the bulk states by

$$
f(\phi)=\sum_{\alpha=1}^{N} m_{\alpha} h\left(\phi_{\alpha}\right),
$$

where $m_{\alpha}, \alpha=1, \ldots, N$ are constant factors related to the forces driving the phase transition. The function $h\left(\phi_{\alpha}\right)$ interpolates the energy densities between the bulk phases. For the simulations in Sec. IV, we chose a third order polynomial of the form $h\left(\phi_{\alpha}\right)=\phi_{\alpha}^{2}\left(3-2 \phi_{\alpha}\right)$.

\section{B. Model extensions to include phases with preserved volume fractions}

In the following, the phase-field formalism is extended to situations in which a subset of order parameters has preserved volume. Without loss of generality, we assume the first $A \leqslant N$ physical states $\phi_{\alpha}, \alpha=1, \ldots, A$ to be subject of volume constraints. The states $\phi_{\alpha}, \alpha=A+1, \ldots, N$ remain to be nonconserved order parameters and hence may undergo phase transitions or grain coarsening processes.

To establish volume conservation of individual ordering states $\phi_{\alpha}, \alpha=1, \ldots, A$, we propose an additional bulk energy density contribution $g(\phi)$ to the functional $\mathcal{F}(\phi)$ in Eq. (1) of the form

$$
g(\phi)=\sum_{\alpha=1}^{A} \chi_{\alpha} h\left(\phi_{\alpha}\right)=\sum_{\alpha=1}^{N} \chi_{\alpha} h\left(\phi_{\alpha}\right)
$$

where the $\chi_{\alpha}:=0$ for $\alpha=A+1, \ldots, N$ have only been introduced in order to abbreviate the following formulas. The idea is to choose the $\chi_{\alpha}=\chi_{\alpha}(t), \alpha=1, \ldots, A$, in such a way that volume changes of the corresponding states $\phi_{\alpha}$ due to the other terms in Eq. (2) are counterbalanced. The energy density functional including the function $g(\phi)$ for volume preservation reads

$$
\mathcal{F}(\phi)=\int_{\Omega}\left(\varepsilon a(\phi, \nabla \phi)+\frac{1}{\varepsilon} w(\phi)+f(\phi)+g(\phi)\right) d x .
$$


With the additional energy contribution (7) the extended set of phase-field equations with preserved volumes can be rewritten as

$$
\tau \varepsilon \partial_{t} \phi_{\alpha}=\mathrm{rhs}_{\alpha}-\lambda-g_{, \phi_{\alpha}}(\phi)-\Lambda, \quad \alpha=1, \ldots, N,
$$

where the term

$$
\Lambda=-\frac{1}{N} \sum_{\alpha=1}^{N} \chi_{\alpha} h_{, \phi_{\alpha}}\left(\phi_{\alpha}\right)
$$

is an additional contribution to the Lagrange multiplier to ensure the constraint $\sum_{\alpha=1}^{N} \phi_{\alpha}(\vec{x}, t)=1$.

The precise choice of the $\chi_{\alpha}(t), \alpha=1, \ldots, A$ will be discussed below. Let us first give an interpretation of

$$
g_{, \phi_{\alpha}}(\phi)=\chi_{\alpha} h_{, \phi_{\alpha}}\left(\phi_{\alpha}\right) \text {. }
$$

Since the derivative of the interpolation function $h_{, \phi_{\alpha}}\left(\phi_{\alpha}\right)$ is nonzero only in the diffuse interface region, the effect of the correction term Eq. (11) is a redistribution of material along the boundaries of phase $\alpha$. To guarantee the summation constraint of the set of phase fields $\phi$, the new redistribution force is accompanied by an additional Lagrange multiplier term $\Lambda$ given by Eq. (10).

The terms rhs ${ }_{\alpha}$ in Eq. (9) can be considered as forces acting on the boundary of the phase for which the order parameter $\phi_{\alpha}$ stands (see $[9,12]$ ). They consist of surface forces [contributions from the potentials $a(\phi, \nabla \phi)$ and $w(\phi)]$ and bulk forces [contribution from $f(\phi)$ ]. In the pure gradient flow dynamics (2) these forces may change the volume occupied by the phase $\alpha$. The term (11) will avoid a volume change and may therefore be interpreted as an antiforce. Since $\chi_{\alpha}=\chi_{\alpha}(t)$ is a function of time only this antiforce is equally distributed along the phase boundary of phase $\alpha$. In particular, it is independent of the neighboring phases. The phase boundary can move but only in such a way that a local movement into the domain occupied by phase $\alpha$, which leads to a loss of its total volume, is counterbalanced by a local phase boundary movement out of the domain somewhere else. This can also be seen as some kind of redistribution, whence Eq. (11) may also be called redistribution term and Eq. (7) redistribution energy.

Let us now discuss the choice of the coefficients $\chi_{\alpha}, \alpha$ $=1, \ldots, A$. For an ordering state being a conserved quantity, the integral of the respected order parameter $\phi_{\alpha}$ over the entire volume $\Omega$ is a constant $V_{\alpha}$. This constant is identical to the initial volume $V_{\alpha}^{0}$ of the corresponding state

$$
\int_{\Omega} \phi_{\alpha}(\vec{x}, t) d \vec{x}=V_{\alpha}(t)=\mathrm{const}\left(=\int_{\Omega} \phi_{\alpha}^{0}(x) d \vec{x}=V_{\alpha}^{0}\right) .
$$

The time derivative of the constant volume $V_{\alpha}$ vanishes. Using Eqs. (9), (3), and (10)

$$
\begin{aligned}
0= & \int_{\Omega} \partial_{t} \phi_{\alpha}(\vec{x}, t) d \vec{x} \\
= & \frac{1}{\tau \varepsilon} \int_{\Omega}\left(\operatorname{rhs}_{\alpha}(\vec{x}, t)-\frac{1}{N} \sum_{\beta=1}^{N} \operatorname{rhs}_{\beta}(\vec{x}, t)-\chi_{\alpha}(t)\right. \\
& \left.\times h_{, \phi_{\alpha}}\left[\phi_{\alpha}(\vec{x}, t)\right]+\frac{1}{N} \sum_{\beta=1}^{N} \chi_{\beta}(t) h_{, \phi_{\beta}}\left[\phi_{\beta}(\vec{x}, t)\right]\right) d \vec{x} .
\end{aligned}
$$

Introducing the abbreviations

$$
R_{\alpha}(t):=\int_{\Omega} \operatorname{rhs}_{\alpha}(\vec{x}, t) d \vec{x} \quad \text { and } H_{\alpha}(t):=\int_{\Omega} h_{, \phi_{\alpha}}\left[\phi_{\alpha}(\vec{x}, t)\right] d \vec{x},
$$

we obtain the set of conditions

$$
\begin{aligned}
0= & R_{\alpha}(t)-\frac{1}{N} \sum_{\beta=1}^{N} R_{\beta}(t)-H_{\alpha}(t) \chi_{\alpha}(t) \\
& +\frac{1}{N} \sum_{\beta=1}^{N} H_{\beta}(t) \chi_{\beta}(t) \quad \text { for } \alpha=1, \ldots, A
\end{aligned}
$$

to determine the $\chi_{\alpha}, \alpha=1, \ldots, A$. Recall that $\chi_{\alpha}=0$ for $\alpha$ $=A+1, \ldots, N$.

Subtracting Eq. (15) for some $\alpha \leqslant A$ from Eq. (15) for another index $\beta \neq \alpha, \beta \leqslant A$ gives $H_{\beta} \chi_{\beta}=H_{\alpha} \chi_{\alpha}+\widetilde{R}_{\beta}-\widetilde{R}_{\alpha}$ where $\widetilde{R}_{\alpha}:=R_{\alpha}-\frac{1}{N} \sum_{\beta=1}^{N} R_{\beta}$. Inserting this identity for $H_{\beta} \chi_{\beta}$ into the last term of Eq. (15) we infer that

$$
\begin{aligned}
\chi_{\alpha}(t)= & \frac{1}{H_{\alpha}(t)}\left(\widetilde{R}_{\alpha}(t)+\frac{1}{N-A} \sum_{\beta=1}^{A} \widetilde{R}_{\beta}(t)\right) \\
& \text { for } \alpha=1, \ldots, A \text { in the case } A<N .
\end{aligned}
$$

In the other case $A=N$ one of the constraints is redundant. Indeed, if the volumes of $N-1$ phases are preserved then, clearly, also the volume of the remaining phase is preserved. Hence also the $\chi_{\alpha}, \alpha=1, \ldots, N$ are not uniquely determined. But instead of proceeding as in the case $A=N-1$ we prefer the following computationally somewhat cheaper choice [of which we can see directly from Eq. (15) that it does the job]:

$$
\chi_{\alpha}(t):=\frac{R_{\alpha}(t)}{H_{\alpha}(t)} \quad \text { for } \alpha=1, \ldots, N \text { in the case } A=N .
$$

\section{NUMERICAL SOLVING ALGORITHM}

A finite difference method on a uniform rectangular mesh with an explicit time marching scheme is used to numerically solve the set of phase-field equations [Eq. (9)]. We denote the time iteration by $n$ with $n=0, \ldots, N_{t}$ and the space coordinates by $i, j, k$ with $i=0, \ldots, N_{x}, j=0, \ldots, N_{y}$, and $k$ $=0, \ldots, N_{z}$.

The numerical solving algorithm consists of two steps: The first part contains a time evolution step of the basic phase-field model [Eq. (2)] resulting in an intermediate state 
of the order parameters $\phi_{\alpha}$. The second step updates the intermediate state, so that the volume fractions of the respective phases are preserved and the final time update of the order parameter is obtained. We use the wide tilde $\widetilde{\phi}_{\alpha}$ notation to indicate an intermediate state of the order parameter $\phi_{\alpha}$ after a time step of the basic phase-field equations in Eq. (2) is done and before the volume change is redistributed by the execution of the suitable antiforcing term in Eq. (7).

The discrete time evolution of Eq. (2) to update the intermediate order parameter $\widetilde{\phi_{\alpha}}$ reads

$$
\left.\widetilde{\left(\phi_{\alpha}\right.}\right)_{i, j, k}^{n+1}=\left(\phi_{\alpha}\right)_{i, j, k}^{n}+\frac{\Delta t}{\tau \varepsilon}\left[\left(\mathrm{rhs}_{\alpha}\right)_{i, j, k}^{n}-\lambda_{i, j, k}^{n}\right],
$$

where $\Delta t$ is an appropriate time step ensuring the stability of the explicit method. For the spatial derivatives in $\left(\mathrm{rhs}_{\alpha}\right)_{i, j, k}^{n}$, we apply forward and backward differences for the divergence of the flux $\nabla^{l} \cdot a_{, \nabla \phi_{\alpha}}\left(\phi, \nabla^{r} \phi\right)$ and centered differences for the term $a_{, \phi_{\alpha}}\left(\phi, \nabla^{c} \phi\right)$. By substituting a sum over all grid points for the integral formulation in Eq. (12), the initial volume $V_{\alpha}^{0}$ and the volume $V_{\alpha}^{n}$ of the physical state $\phi_{\alpha}$ at time $n$ in the discrete computational domain $\Omega$ can be written as

$$
V_{\alpha}^{0}=(\Delta x)^{3} \sum_{i, j, k}\left(\phi_{\alpha}\right)_{i, j, k}^{0} \quad \text { and } V_{\alpha}^{n}=(\Delta x)^{3} \sum_{i, j, k}\left(\phi_{\alpha}\right)_{i, j, k}^{n} .
$$

We introduce the short notation

$$
\left(R_{\alpha}\right)_{i, j, k}^{n+1}=\left(\mathrm{rhs}_{\alpha}\right)_{i, j, k}^{n}-\lambda_{i, j, k}^{n}
$$

in order to rewrite the local change of the physical state $\phi_{\alpha}$ at a grid point $(i, j, k)$ from time $n$ to $n+1$,

$$
\left[\left(\phi_{\alpha}\right)_{i, j, k}^{n+1}-\left(\phi_{\alpha}\right)_{i, j, k}^{n}\right]=\frac{\Delta t}{\tau \varepsilon}\left[\left(R_{\alpha}\right)_{i, j, k}^{n+1}\right] .
$$

Volume preservation is achieved numerically by computing the antiforce $g_{, \phi_{\alpha}}(\phi)$ in Eq. (11) and the corresponding Lagrange multiplier $\Lambda$ in Eq. (10) in a discrete form and as a function of the intermediate states $\widetilde{\phi_{\alpha}}$,

$$
g_{, \phi_{\alpha}}(\widetilde{\phi})_{i, j, k}^{n+1}=\chi_{\alpha}^{n+1} h_{, \phi_{\alpha}}\left[\left(\widetilde{\phi_{\alpha}}\right)_{i, j, k}^{n+1}\right]
$$

and

$$
\Lambda_{i, j, k}^{n+1}=-\frac{1}{N} \sum_{\beta=1}^{N} \chi_{\beta}^{n+1} h_{, \phi_{\beta}}\left[\left(\widetilde{\phi_{\beta}}\right)_{i, j, k}^{n+1}\right] .
$$

Combining Eqs. (14) and (21) gives

$$
\begin{gathered}
\left.R_{\alpha}^{n+1}=(\Delta x)^{3} \sum_{i, j, k}\left(R_{\alpha}\right)_{i, j, k}^{n+1}=\frac{\tau \varepsilon(\Delta x)^{3}}{\Delta t} \sum_{i, j, k}\left[\widetilde{\left(\phi_{\alpha}\right.}\right)_{i, j, k}^{n+1}-\left(\phi_{\alpha}\right)_{i, j, k}^{n}\right] \\
\left.H_{\alpha}^{n+1}=(\Delta x)^{3} \sum_{i, j, k} h_{, \phi_{\alpha}}\left[\widetilde{\left(\phi_{\alpha}\right.}\right)_{i, j, k}^{n+1}\right] .
\end{gathered}
$$

By inserting these relations into Eq. (16), the values of the counterbalance force $\chi_{\alpha}^{n+1}$ can be computed. The time update of the order parameter $\left(\phi_{\alpha}\right)_{i, j, k}^{n+1}$ at time $n+1$ ensuring volume preservation of the respective physical state is finalized by adding the redistribution force and the associated Lagrange multiplier in Eq. (22) with appropriate parameters $\frac{\Delta t}{\tau \varepsilon} \chi_{\alpha}^{n+1}$ to the intermediate state $\left(\phi_{\alpha}\right)_{i, j, k}^{n+1}$, i.e.,

$$
\left(\phi_{\alpha}\right)_{i, j, k}^{n+1}=\left(\widetilde{\phi}_{\alpha}\right)_{i, j, k}^{n+1}-\frac{\Delta t}{\tau \varepsilon} \chi_{\alpha}^{n+1} h_{, \phi_{\alpha}}\left[{\widetilde{\left(\phi_{\alpha}\right.}}_{i, j, k}^{n+1}\right]-\frac{\Delta t}{\tau \varepsilon} \Lambda_{i, j, k}^{n+1} .
$$

Since the initial volume of the physical state is preserved, i.e., $V_{\alpha}^{n}=V_{\alpha}^{0}$, we can exploit the simplification of Eq. (23),

$$
\begin{aligned}
\left.\sum_{i, j, k}\left[\widetilde{\left(\phi_{\alpha}\right.}\right)_{i, j, k}^{n+1}-\left(\phi_{\alpha}\right)_{i, j, k}^{n}\right] & \left.=\sum_{i, j, k} \widetilde{\left(\phi_{\alpha}\right.}\right)_{i, j, k}^{n+1}-\sum_{i, j, k}\left(\phi_{\alpha}\right)_{i, j, k}^{n} \\
& \left.=\sum_{i, j, k} \widetilde{\left(\phi_{\alpha}\right.}\right)_{i, j, k}^{n+1}-V_{\alpha}^{0},
\end{aligned}
$$

in our numerical scheme.

\section{SIMULATION APPLICATIONS}

\section{A. Bubble clusters}

To demonstrate the capability of the volume preserving multiphase-field method, we first study the evolution of bubble clusters driven by surface energy minimization. Since thereby no changes in volume of the individual bubbles are possible, an incompressibility of the bubble interior is assumed. This is a valid approximation, when the volume work to change the individual bubble size is high compared to the energy stored in its surface [14]. Also, since the curvature driven process is slow compared to the reorganization of fluid particles in the interior or exterior of the bubbles, we can neglect any fluid dynamics.

In the simulations, four phase-field parameters were used for the bubbles and one for the surrounding matrix phase, each of them bearing a volume constraint. As in the other simulations presented in the following, the bubbles were first filled into the regular simulation grid with a sharp transition from 0 to 1 . Subsequently a simple numerical diffusion algorithm was applied to produce smooth profiles over a distance of the interface parameter $\varepsilon$ (all simulation parameters are given in the figure captions). In the first simulation run, a quadruple bubble ensemble was chosen as the initial configuration in the form of four adjacent cubes, as shown in Fig. 1(a). The quadruple line in the center is energetically instable and splits up into two triple lines, as expected. Using the same volumes as before, a different initial setting with four adjacent spheroidal bubbles, aligned in a zig-zag manner along the space diagonal of the box, was used [Fig. 1(b)]. Both initial settings lead to the same standard quadruple bubble, which is given in Fig. 1(c) as a result of the second run. In the images, the $\phi_{\alpha}=0.5$ isosurfaces of the four bubble phase fields are visualized.

The corresponding structural dynamics is reflected in the surface energy computed from the phase-field data according to Eq. (1), illustrated in Fig. 2. The plateau in the energy evolution of the second run (dotted curve) is caused by the preliminary formation of a triple bubble cluster with a fourth 
a)

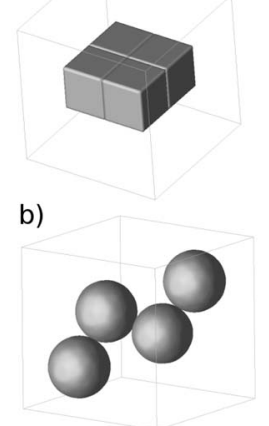

c)

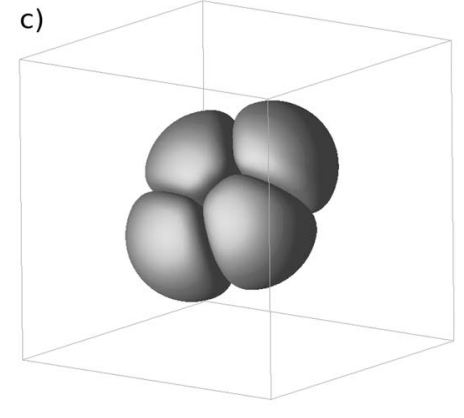

d)

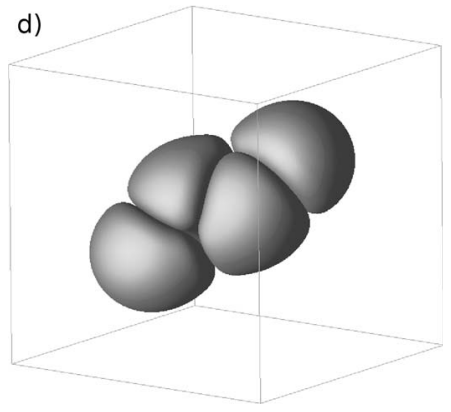

FIG. 1. (a), (b) Two different initial configurations of four bubbles in a matrix phase, both leading to the final standard quadruple bubble (c). An intermediate state of the cluster starting from condition (b) is given in (d). For the simulations, a box of $75 \times 75 \times 75$ grid points was used with a regular grid spacing of $\Delta x=\Delta y=\Delta z=0.02$, an interface width $\varepsilon=0.1$, and time constant of $\Delta t=3 \times 10^{-5}$. The parameter of the higher order terms in Eq. (5) was set $\gamma_{\alpha \beta \delta}=6.0$ and the surface energies of the bubble boundaries are $\gamma_{\alpha \beta}=1.0$.

outer neighbor bubble on the top right, as depicted in Fig. $1(d)$.

\section{B. Double crystals}

As a further application of the phase-field model with volume constraints, we study the stability of growth morphologies of faceted cubic double crystals embbeded in a third phase in two dimensions. Various volume ratios $R$ $=V_{B} / V_{A}$ of two crystals $A$ and $B$ are studied in the simulations, always assuming a conserved volume for each of them. This situation is an example of shape evolution under surface diffusion, in the limit when surface diffusion is fast compared to the diffusional mass transport between both crystals by diffusion through the surrounding medium.

We choose anisotropies leading to square Wulff shapes for each of the two crystals (in two dimensions) and name the ratio between the internal crystalline boundary energy and the outer boundary energy by $\lambda$. Under this assumption, it has been proven in [5] that three different types of double crystals exist depending on the value of $\lambda$ and $R$ : A rectangular bicrystal with straight internal boundary (type I), a bigger square crystal in contact with a smaller rectangular one (type II), and a square-shaped bicrystal with the small one sitting at one edge (type III). The geometric dimensions of the equilibrium shape of each double crystal type can be computed by minimizing its surface energy, which is a function of the dimension parameters $x$ and $y$ for types I and II (see insets of Fig. 3). The coexistence lines shown by solid lines in Fig. 3 respectively separate two regions of identical equilibrium morphology (I/II, I/III, and II/III).

The morphology diagram was scanned systematically by performing a series of phase-field simulations for different volume ratios $R$ and different energy ratios $\lambda$. As the initial configuration, always a starting morphology of type II was chosen with nonequilibrium crystal dimensions. Thereby, an identical larger crystal $B$ was set (aspect ratio 1.75) whereas the volume of crystal $A$ was changed to give the desired volume ratio $R$. To realize a facetted habit, a crystalline surface energy density of the form

$$
a(\phi, \nabla \phi)=\sum_{\alpha<\beta} \gamma_{\alpha \beta}\left(\max _{1 \leqslant k \leqslant 4}\left\{\left(\phi_{\alpha} \nabla \phi_{\beta}-\phi_{\beta} \nabla \phi_{\alpha}\right) \cdot \vec{\eta}_{k}\right\}\right)^{2}
$$

was used, where $\eta_{k}, k=1, \ldots, 4$ denote four unit edge vectors of a square Wulff form. The edge vectors were chosen in diagonal direction of the coordinate system leading to the development of horizontal and vertical phase boundaries in the simulation box. In Fig. 3, the resulting morphology types for discrete values of $R$ and $\lambda$ are indicated by symbols. Each simulation was continued long enough to ensure stationarity ( $\approx 7 \times 10^{5}$ time steps). The morphology boundaries I/II and I/III are resolved well by the simulations, whereas the transition from type II to type III is shifted to larger volume ratios $R$. We attribute this behavior to the existence of an energy barrier (possibly related to the diffuse interface formulation), since extended simulation studies in the coexist-

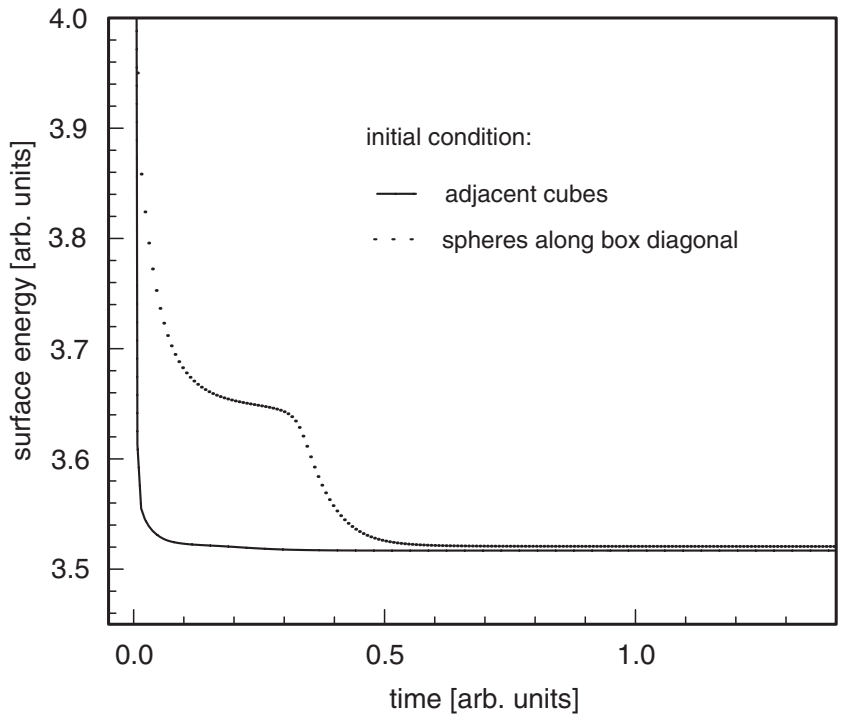

FIG. 2. Plot of the surface energy evolution for the quadruple bubble simulations with two different starting configurations. The step appearing in the curve for the four spherical bubble results from the preliminiary configuration given in Fig. 1(d). 


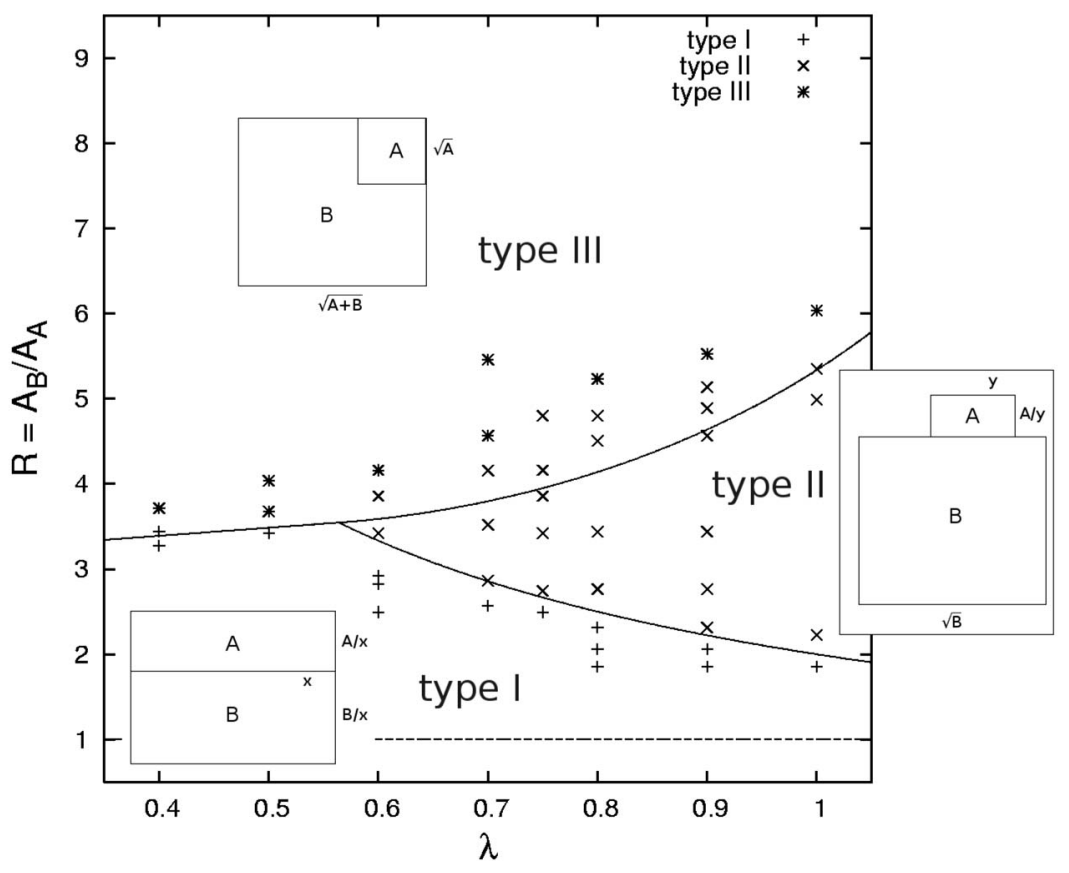

FIG. 3. Morphology diagram of the double crystal system with coexistence lines (solid) between different crystal types (insets). The final morphologies found in the phase-field simulations are indicated as symbols referring to the different types. The computational parameters were $200 \times 200$ grid points, $\Delta t=2.0 \times 10^{-5}, \quad \gamma_{\alpha \beta}=1.0$ for the outer boundary of both crystals, and $\lambda \in[0.4,1]$ for the double crystal interface.

ence region have shown a dependence of the final structure type on the initial condition. A detailed discussion of the best choice of initial simulation configuration and an extension to a three-dimensional (3D) morphology selection of double crystals is in preparation [15].

\section{Grain growth with second-phase contributions}

Grain growth, a surface curvature driven process leading to a gradual coarsening of a polycrystalline structure, involves the dissolution and growth of individual grains by grain boundary movement. Many alloys contain inert precipitates of a second phase which may decisively reduce the coarsening process by pinning of the grain boundaries, an effect known as Zener pinning and first described in [16]. As a simplified example of this problem, we investigated grain coarsening in the presence of second phase particles, represented by phase-field variables with constrained volumes. To create an initial situation, a random tesellation of the $2 \mathrm{D}$ computational domain into 250 cells was carried out. Thereby, Voronoi partitioning was applied, an algorithm comparably used in the construction of the Wigner-Seitz cell in solid-state physics. The result is shown in Fig. 4(a), where the quantity $\Sigma_{\alpha>\beta} \phi_{\alpha} \phi_{\beta}$ is plotted to indicate the diffuse interfaces. Volume conservation was applied to $13 \%$ of the grains, marked in gray in Figs. 4(a) and 4(b). In Fig. 4(b), a snapshot of the grain boundaries after 16000 time steps is displayed when the coarsening of the structure is evident. The second-phase grains tend to form linear clusters and adapt their shape according to minimize curvature [to model the influence of a predominant particle shape, a surface energy anisotropy as given in Eq. (26) can be used for the boundaries between normal and inert phase grains].

If, on the other hand, the volume of all phase fields is preserved, the system behaves like an incompressible polyhedral foam. The result is given in Fig. 4(c). As can be seen from the comparison with the initial situation [Fig. 4(a)],

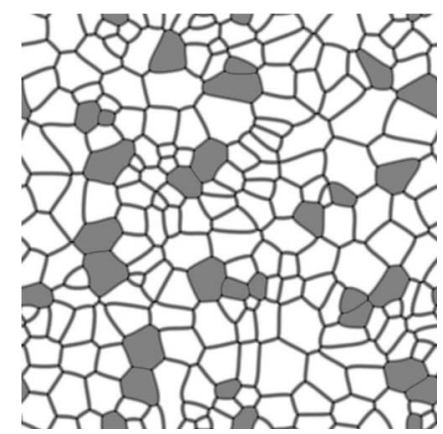

(a)

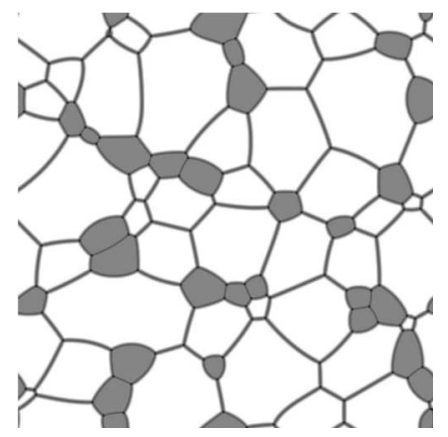

(b)

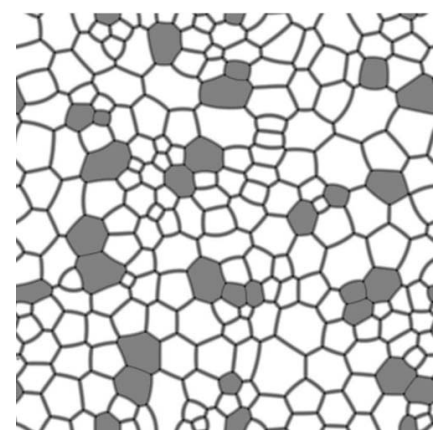

(c)

FIG. 4. (a) Initial configuration of a polycrystal produced by a Voronoi tesselation of the domain. The simulation results after 16000 time steps for (b) a structure with $13 \%$ of the grains with preserved volumes (marked in gray) is compared with a foamlike structure in (c), where cell volumes are preserved. The computational parameters were $400 \times 400$ grid points, $\Delta x=\Delta y=\Delta z=1.0, \Delta t=0.8, \varepsilon=3.0, \gamma_{\alpha \beta}=0.25$ for all grain boundaries and $\gamma_{\alpha \beta \delta}=3.0$. 
only minor rearragements of the bubbles take place, which are accompanied by a collective movement of their surroundings.

\section{CONCLUSION}

In conclusion, we have introduced a different phase-field model that allows one to treat pattern formations and microstructure evolutions in multiphase systems in which a subset of phases obeys volume constraints. Bubble clusters develop into an equilibrium configuration of minimal surface energy and the computational analysis of double crystal structures is consistent with theoretical predictions. The application to foam structures demonstrates the ability of the method to consider grain growth in the presence of phases with preserved volume fractions. It is expected that the presented phase-field model opens a broad range of new applications such as, i.e., the study of inert particles in phase transformation processes, the inclusion of partial melts or bubbles in textures, and the role of nucleation sides.
[1] A. Elmoutaouakkil, L. Salvo, E. Maire, and G. Peix, Adv. Eng. Mater. 4, 803 (2002).

[2] F. Graner, Y. Jiang, E. Janiaud, and C. Flament, Phys. Rev. E 63, 011402 (2000).

[3] D. Weaire, Phys. Scr. T45, 29 (1992).

[4] C. Carter, A. Roosen, J. Cahn, and J. E. Taylor, Acta Metall. Mater. 43, 4309 (1995).

[5] B. Wecht, M. Barber, and J. Tice, Acta Crystallogr., Sect. A: Found. Crystallogr. A56, 92 (2000).

[6] B. Evans, J. Renner, and G. Hirth, Intern. J. Earth Sc. 90, 88 (2001).

[7] J. Renner, B. Evans, and G. Hirth, Contrib. Mineral. Petrol. 142, 501 (2002).

[8] N. Walte, J. Becker, P. Bons, D. Rubie, and D. Frost, Earth Planet. Sci. Lett. 262(3-4), 517 (2007).
[9] H. Garcke, B. Nestler, and B. Stoth, SIAM J. Appl. Math. 60, 295 (1999).

[10] B. Nestler, H. Garcke, and B. Stinner, Phys. Rev. E 71, 041609 (2005).

[11] B. Stinner, in Free Boundary Problems, International Series on Numerical Mathematics No. 154 (Birkhäuser, 2007), pp. 413423.

[12] H. Garcke, B. Stoth, and B. Nestler, Interfaces Free Boundaries 1, 175 (1999).

[13] X. Yang, J. J. Feng, C. Liu, and J. Shen, J. Comput. Phys. 218, 417 (2006).

[14] H. Aref and D. L. Vainchtein, Phys. Fluids 12, 23 (2000).

[15] Subject to a comprehensive study in a forthcoming paper.

[16] C. S. Smith, Trans. Am. Inst. Min., Metall. Pet. Eng. 175, 47 (1948). 